\title{
Métodos electroquímicos semicuantitativos de estudio de la corrosión por picaduras del acero para armaduras de hormigón*
}

M. C. ANDRADE** y J. A. GONZALEZ***

\section{N T R O D U C C I O N}

El acero embebido en el hormigón se encuentra sometido a un medio de elevada alcalinidad ( $\mathrm{pH}$ comprendido entre 12 y 13), debido a la cal libre, a los álcalis que contiene el cemento, y al $\mathrm{Ca}(\mathrm{OH})_{2}$ que se forma durante la hidratación de los silicatos. En esta situación, el acero se halla recubierto por una capa de óxidos e hidróxidos estables que lo mantienen pasivado, interponiendo una barrera entre él y los agentes agresivos.

No obstante, bajo ciertas circunstancias, las armaduras embebidas en hormigón se pueden corroer, siendo dos las causas del ataque fundamentalmente: a) Una disminución del $\mathrm{pH}$ (debida, por ejemplo, a la carbonatación ıdel hormigón) que hace desaparecer la capa de pasivación, dando lugar a una corrosión generalizada. b) La presencia de agentes despasivantes en la masa del hormigón, que provoca una corrosión localizada en puntos aislados.

De estas dos formas de corrosión del acero la más grave, por sus consecuencias es la segunda. El ataque suele adoptar la forma de picaduras, cuyo fondo es anódico respecto al resto de la superficie metálica, por lo que progresa en profundidad (1).

A pesar de haberse realizado numerosos estudios para esclarecerlo (2-5), no se conoce aún el mecanismo concreto del ataque por picaduras. En lo que coinciden todos los autores, es en señalar al ion $\mathrm{Cl}^{-}$como el agente despasivante más peligroso; $\mathrm{y}$, dada la frecuencia con que dicho ion puede estar presente en la masa del hormigón (bien porque se le añada como aditivo para mejorar algunas de sus propiedades físico-químicas, o porque penetre a través de la red de poros, al estar situada la estructura en ambiente marino), ha parecido oportuno profundizar en el estudio de técnicas de medida capaces de detectar posibles deterioros locales de la capa de pasivación, ya sean producidos por los cloruros u otros agentes despasivantes, como los sulfuros, sulfatos, etc. Pues este aspecto parece tan interesante como la determinación cuantitativa de la velocidad de corrosión, en todos aquellos casos en que, como en el presente, se desconoce la magnitud del área sobre la que se centra el ataque. Esta consideración cobra especial importancia cuando el metal objeto de observación no es directamente visible, como puede ser el caso de armaduras o la superficie interna de las tuberías, ya que entonces, como paso previo a una cuantificación del ataque, se necesita conocer su morfología (si es uniforme, por picaduras, intergranular ...).

* Ante el interés demostrado por algunos lectores se publica este trabajo, que es previo al presentado por los mismos autores en el n.o 165 de esta Revista.

* C. Andrade. Dra. en Quím. Ind. Colabora actualmente con los Departamentos de Corrosión y Protección del CENIM y de Química del IETcc.

*** J. A. González, Dr. en Quím. Ind. Inv. Ci€nt. del Departainento de Corrosión y Protección del CENIM. 
Son muchos los estudios existentes sobre los métodos electroquímicos de medida de la corrosión generalizada (6) (7) (8); incluso los autores del presente trabajo han estudiado ya su aplicación al caso de probetas de hormigón armado (9), encontrando que el de "resistencia de polarización" (6) (8) es el más prometedor de todos ellos.

También son numerosos los trabajos publicados sobre la detección de corrosión localizada mediante técnicas eloctroquímicas (10-14), habiéndose llevado a cabo revisiones bibliográficas muy completas al respecto (15) (16) (17). Tal detección se basa en el hecho de que el ataque localizado se puede definir por el valor del potencial de electrodo al que se produce; es decir, existen unos potenciales críticos de "picado" característicos de cada proceso de corrosión por picaduras. Estos potenciales reciben diversos nombres en la bibliografía: "breakthrough potential", "pitting potential", "lochfrass potential..." y sobre su determinación y significado se han aportado ya datos muy valiosos (10) (11) (12) (18).

La figura 1, que reproduce el trazado típico de una curva de polarización potenciocinética, ilustra esquemáticamente el comportamiento del acero en medios alcalinos, con y sin cloruros, señalando los potenciales críticos que delimitan las diferentes regiones en las que se produce corrosión por picaduras, pasiväción imperfecta, pasivación perfecta e inmunidad. La curva a trazos que corresponde a un medio sin cloruros, pone de manifiesto que, en tales circunstancias, se pasa directamente de la pasividad perfecta a la transpasividad, evidenciada por el desprendimiento de oxígeno a unos $+600 \mathrm{mV}$ con respecto al electrodo de calomelanos saturado.

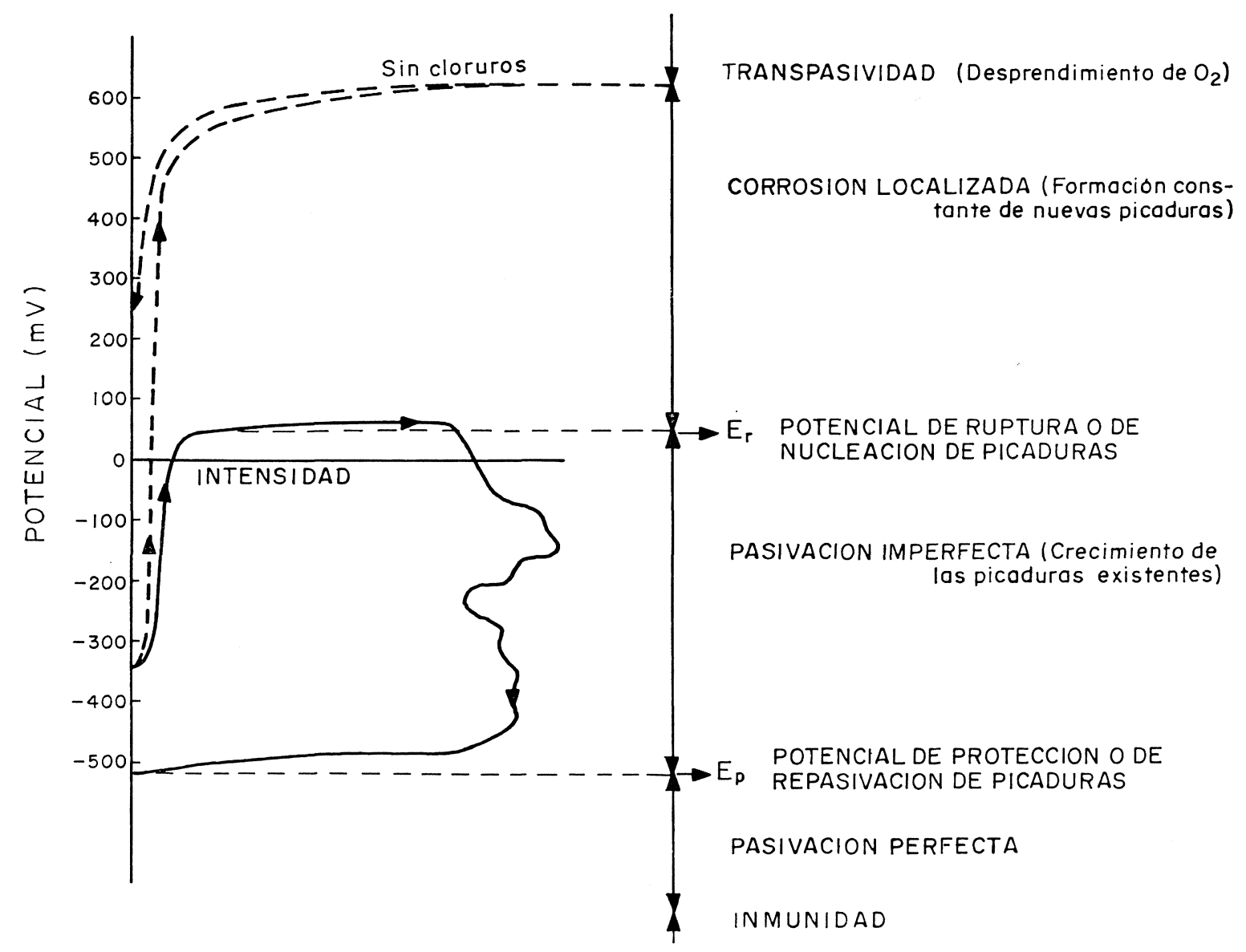

Fig. 1 
Ahora bien, cuando en el medio existen agentes despasivantes, en este caso concreto cloruros, aparece en la rama anódica, por debajo del potencial de desprendimiento de óxigeno, un aumento rápido y progresivo de la intensidad. El potencial al que se produce dicho aumento de intensidad se le denomina "potencial de ruptura" o de "nucleación de picaduras". El aumento brusco de la intensidad, a un potencial dado inferior al de la transpasivación, es indicio de la rotura de la capa de pasivación y de la aparición de picaduras. El punto de corte de la curva de retorno con el eje de ordenadas se le considera como potencial de "repasivación" o de "protección" (3).

En el presente trabajo se pretende, por una parte, contrastar las posibilidades de los métodos electroquímicos de detección del ataque localizado en el caso concreto de las armaduras del hormigón y, por otra, comprobar el efecto despasivante de los iones cloruro sobre el acero y, asimismo, el posible efecto inhibidor de los iones nitrito, a través de la medida de los potenciales críticos de picado para cada conjunto de condiciones.

\section{MATERIALES Y METODOS EXPERIMENTALES}

Como electrólito en la realización de los ensayos se ha utilizado solución saturada de $\mathrm{Ca}(\mathrm{OH})_{2}$, por ser un medio que reproduce, de una forma aproximada, las condiciones termodinámicas a las que están expuestas las armaduras en el hormigón.

Es importante destacar que la reproducción es "sólo aproximada", ya que:

a) el $\mathrm{pH}$ del $\mathrm{Ca}(\mathrm{OH})_{2}$ saturado es de 12,6 , mientras que la presencia de cationes $\mathrm{Na}^{+}$ $\mathrm{y} \cdot \mathrm{K}^{+}$pueden dar lugar a $\mathrm{pH}$ aún más elevados en el hormigón, en tanto que la adición de $\mathrm{CaCl}_{2}$ u otros aditivos, o la carbonatación, a pH más reducidos;

b) el acceso de oxígeno hasta el acero es completamente diferente que cuando está embebido en hormigón, dado que la movilidad de los iones en disolución es evidentemente muy superior que en el seno de aquél.

Por tanto, aunque las condiciones termodinámicas sean muy similares, la cinética del proceso puede ser muy diferente en ambos medios. De cualquier manera, se ha considerado oportuno realizar estos ensayos en $\mathrm{Ca}(\mathrm{OH})_{2}$ saturado, como paso previo al estudio del problema sobre probetas de hormigón.

Las probetas eran redondas de $7 \mathrm{~mm}$ de diámetro y $8 \mathrm{~cm}$ de longitud, de un acero para construcción de composición:

$\mathrm{C}-0,36 \%, \mathrm{Mn}-0,70 \%, \mathrm{Si}-0,28 \%, \mathrm{P}-0,011 \%, \mathrm{~S}-0,020 \%$ y $\mathrm{Cr}-0,5 \%$.

La superficie sumergida era de $11 \mathrm{~cm}^{2}$, no habiéndose utilizado cinta adhesiva protectora para delimitar la superficie expuesta al ataque, pues se plido comprobar, en coincidencia con otros autores (10), que el ataque por picaduras afectaba preferentemente al metal inmediato a la cinta, falseando el comportamiento de la probeta. Este ataque preferente no se ha constatado en cambio, a pesar de no emplear agitación, en la interfase acero-líquido-aire de los electrodos sumergidos.

Las probetas fueron pulidas con papeles abrasivos de finura creciente hasta el n. ${ }^{\circ} 00$, lavados con agua y desengrasados con acetona antes de ensayarlas.

Como aditivos se utilizaron sustancias con la pureza exigida en los reactivos para análisis. Los cloruros se emplearon en las proporciones de 0,01 y $0,1 \%$, añadiéndolos en forma de $\mathrm{KCl}$, y el efecto del $\mathrm{NaNO}_{2}$ se estudió para las relaciones siguientes:

$$
\begin{array}{lllll}
0,01 & \% & \mathrm{Cl}^{-}+0,01 & \% & \mathrm{NO}_{2}{ }^{-} \\
0,1 & \% & \mathrm{Cl}^{-}+0,1 & \% & \mathrm{NO}_{2}^{-} \\
0,1 & \% & \mathrm{Cl}^{-}+0,2 & \% & \mathrm{NO}_{2}^{-} \\
0,1 & \% & \mathrm{Cl}^{-}+0,5 & \% & \mathrm{NO}_{2}^{-}
\end{array}
$$


Estos porcentajes se refieren siempre al peso de la disolución saturada de $\mathrm{Ca}(\mathrm{OH})_{2}$.

Las medidas electroquímicas se realizaban a los pocos minutos de introducir las probetas en las células, empleando como electrodo auxiliar uno de grafito y, como referencia, el electrodo de calomelanos con disolución saturada de $\mathrm{KCl}$. Al término del ensayo se procedía a la observación visual de la probeta.

Para la determinación de los potenciales críticos de picado se han seguido los métodos potenciocinético, potenciostático y galvanostático. Su fundamento teórico y aplicación a condiciones estrechamente relacionadas con las del trabajo presente ha sido ya objeto de estudios anteriores (10-14) (18).

\section{R E S U L T A D O S}

En la figura 2, obtenida por el método potenciocinético a $50 \mathrm{mV} / \mathrm{min}$, se reproduce el comportamiento del acero en disolución saturada de $\mathrm{Ca}(\mathrm{OH})_{2}$. La línea continua refleja el caso en que no se producen picaduras, que se dan en ausencia de cloruros y, en ocasiones, con $0,01 \% \mathrm{Cl}^{-}$. La línea de trazos muestra el comportamiento típico de una corrosión por picaduras (concentraciones del $0,10 \% \mathrm{Cl}^{-} \mathrm{y}$, en ocasiones, del $0,01 \%$ ).

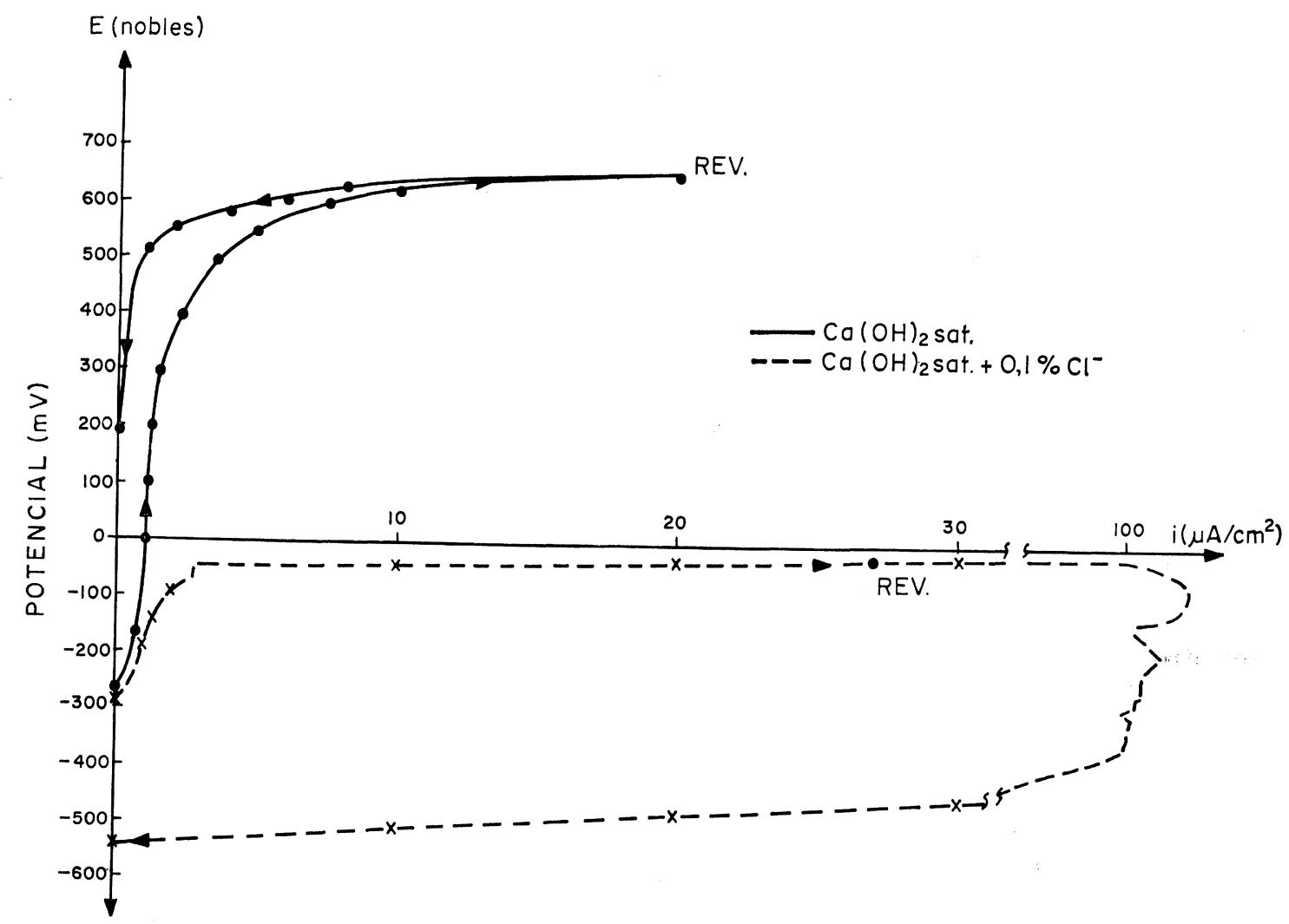

Fig. 2

Los valores del potencial de ruptura, $E_{\mathrm{r}}$, determinados por este método, se hallan sujetos a grandes dispersiones, mientras que los de protección, $E_{\mathrm{p}}$, aparecían siempre a valores muy similares; hecho este, que queda reflejado, para cada conjunto de condiciones, en la tabla I. 


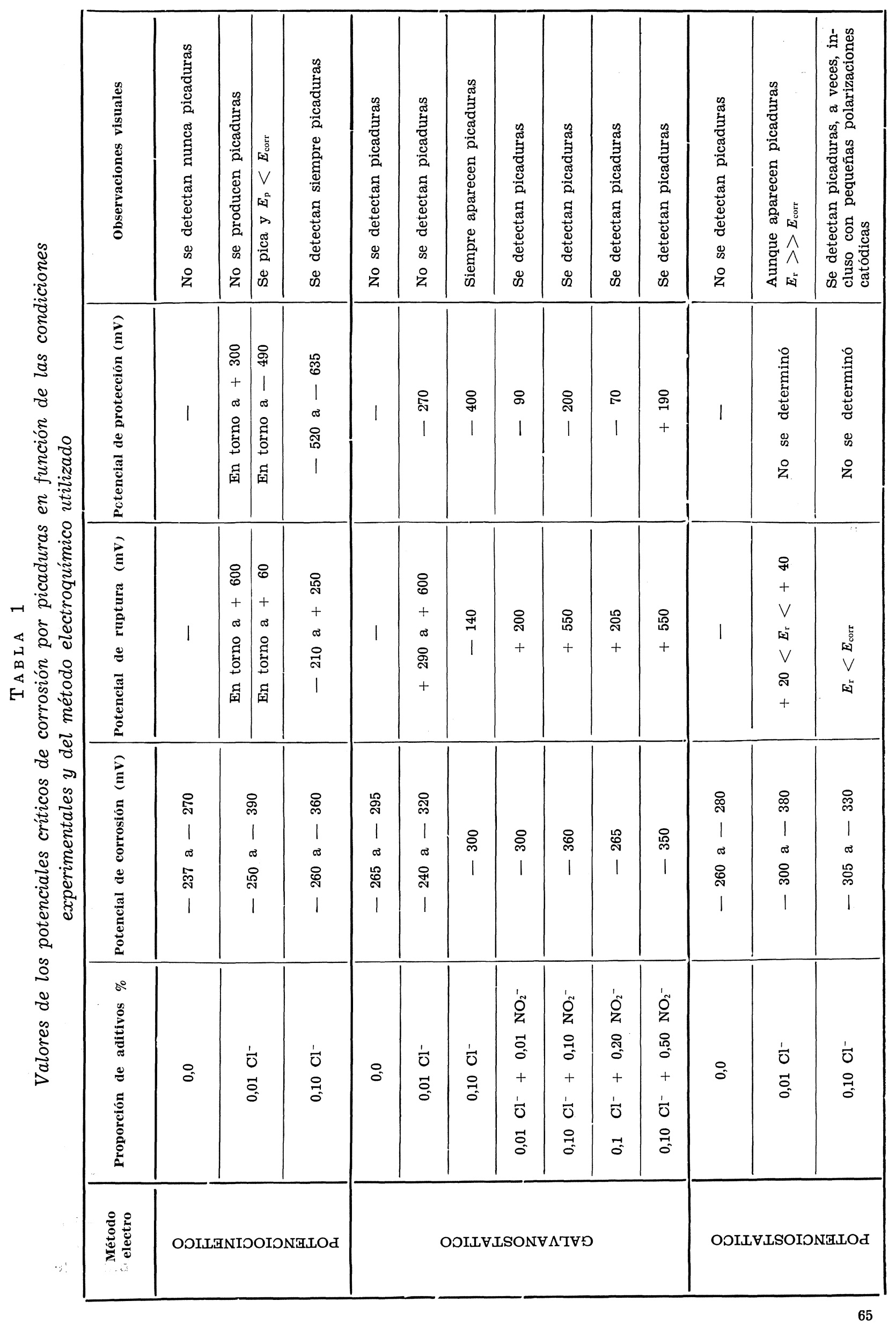


Aunque existen varios factores que pueden afectar los valores de los potenciales críticos, uno de los más importantes es la velocidad de polarización, cuyo efecto sobre el comportamiento de los electrodos en disolución saturada de $\mathrm{Ca}(\mathrm{OH})_{2}$ con un $0,10 \% \mathrm{Cl}^{-}$se recoge en la figura 3 , de la que se deduce una acusada influencia de la citada velocidad sobre los potenciales críticos, lo que hace que el método potenciocinético no resulte apropiado, cuando lo que interesan son valores fiables de $E_{\mathrm{r}} \circ E_{\mathrm{p}}$.

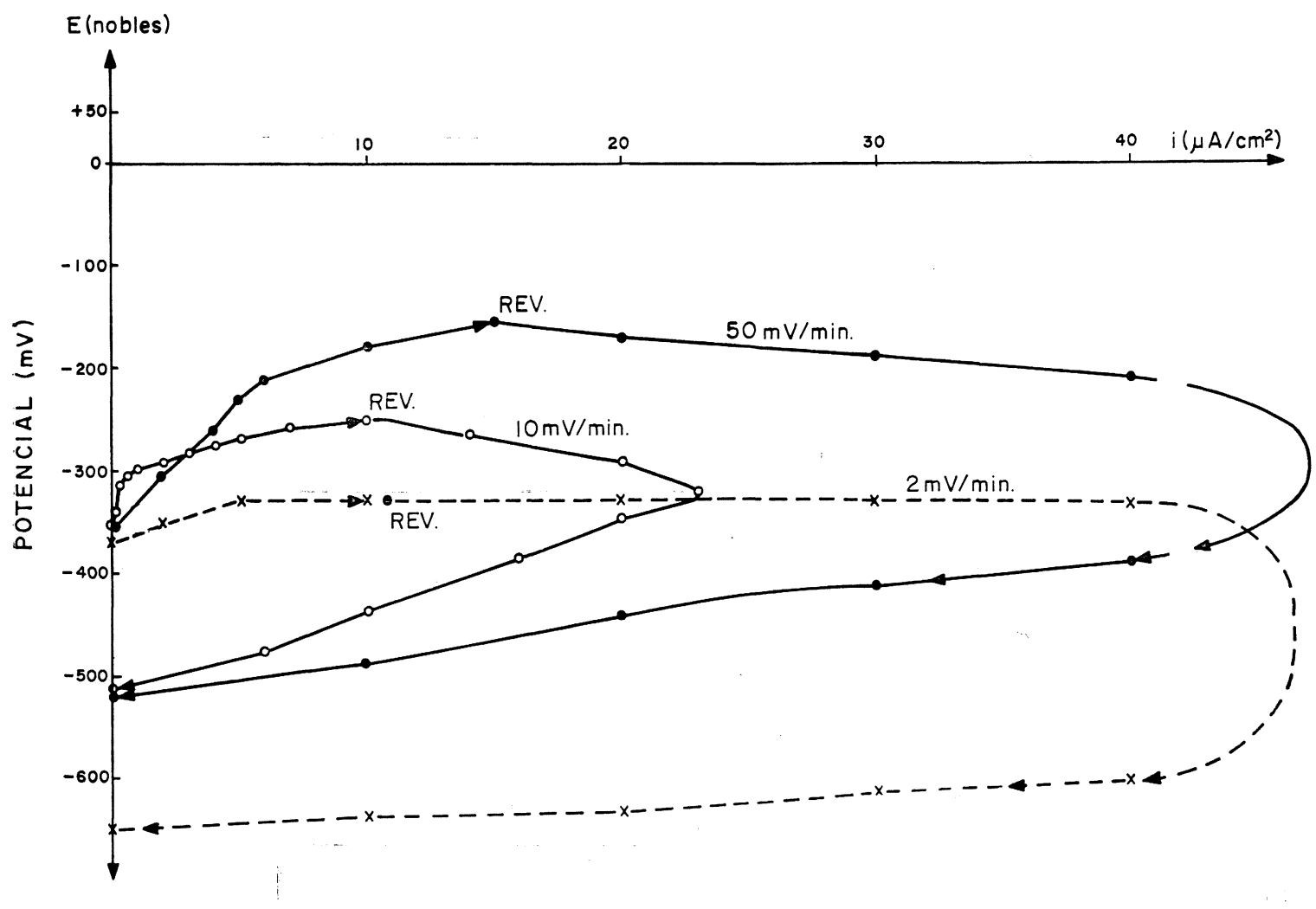

Fig. 3

En la figura 4 se muestra el comportamiento del acero en la solución de $\mathrm{Ca}(\mathrm{OH})_{2}$, sin y con adiciones de $\mathrm{Cl}^{-}$, o de $\mathrm{Cl}^{-}$y $\mathrm{NO}_{2}^{-}$, según las indicaciones del método galvanostático, para una densidad de corriente aplicada de $10 \mu \mathrm{A} / \mathrm{cm}^{2}$. En ausencia de cloruros no se produce rotura de la capa de pasivación, alcanzándose en pocos minutos el potencial de desprendimiento de $\mathrm{O}_{2}$, mientras que, cuando existen en solución un $0,10 \% \mathrm{Cl}^{-}$, tras pasar el potencial por un máximo, inferior al valor correspondiente al desprendimiento de $\mathrm{O}_{2}$, desciende hasta un valor estacionario, situado normalmente en la zona catódica con respecto al potencial de partida $\left(E_{\text {corr }}\right)$. Ciertos autores (15) (17) consideran que el máximo de la curva corresponde al valor de $E_{\mathrm{r}}$ y el valor estacionario final a $E_{\mathrm{p}}$. Se observa, sin embargo, que, como ocurría con el método potenciocinético y se recoge en la tabla I, el primer valor viene afectado por una considerable dispersión, mientras que la que afecta a $E_{\mathrm{p}}$ es mucho menor. Cuando coexisten en solución cloruros y nitritos el comportamiento es similar al que se obtiene cuando sólo existen cloruros, al menos para las condiciones de la figura 4 ; aparece también el máximo que indica la rotura de la capa de pasivación pero, en coincidencia con otros autores (12), se registran unas oscilaciones típicas de la competencia del efecto despasivante de los iones $\mathrm{Cl}^{-}$y el inhibidor de los $\mathrm{NO}_{2}{ }^{-}$. 


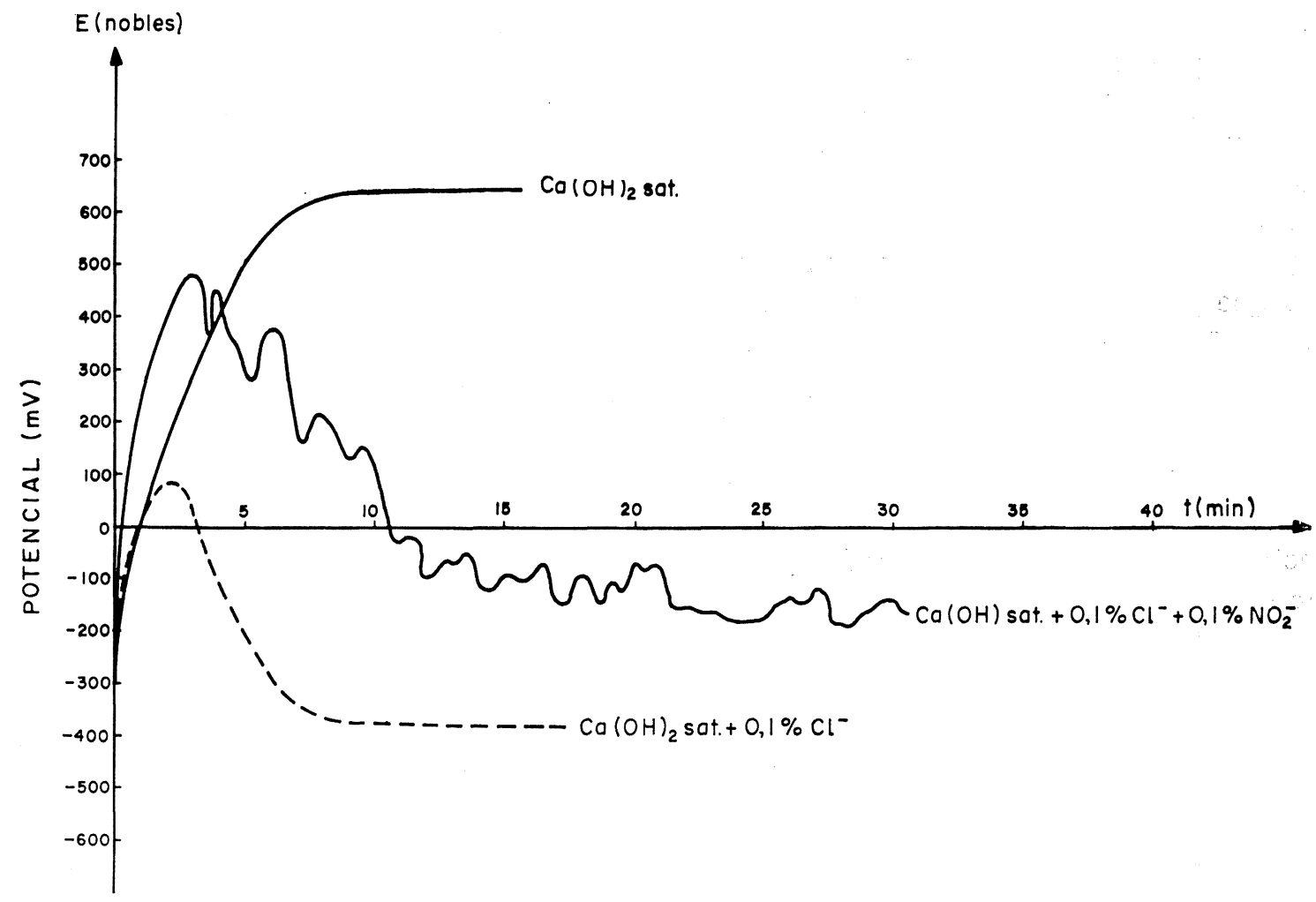

Fig. 4

La figura 5, también relativa al método galvanostático, muestra cómo influye la densidad de corriente aplicada en $E_{\mathrm{r}}$ y $E_{\mathrm{p}}$ para el caso de la disolución saturada de $\mathrm{Ca}(\mathrm{OH})_{2}$ con un $0,10 \% \mathrm{Cl}^{-}$. Se puede apreciar claramente que dicha influencia es muy importante sobre $E_{\mathrm{r}}$ y menos acusada en $E_{\mathrm{p}}$, indicando que tampoco es adecuado el método galvanostático para fijar el valor del potencial de ruptura en el sistema estudiado.

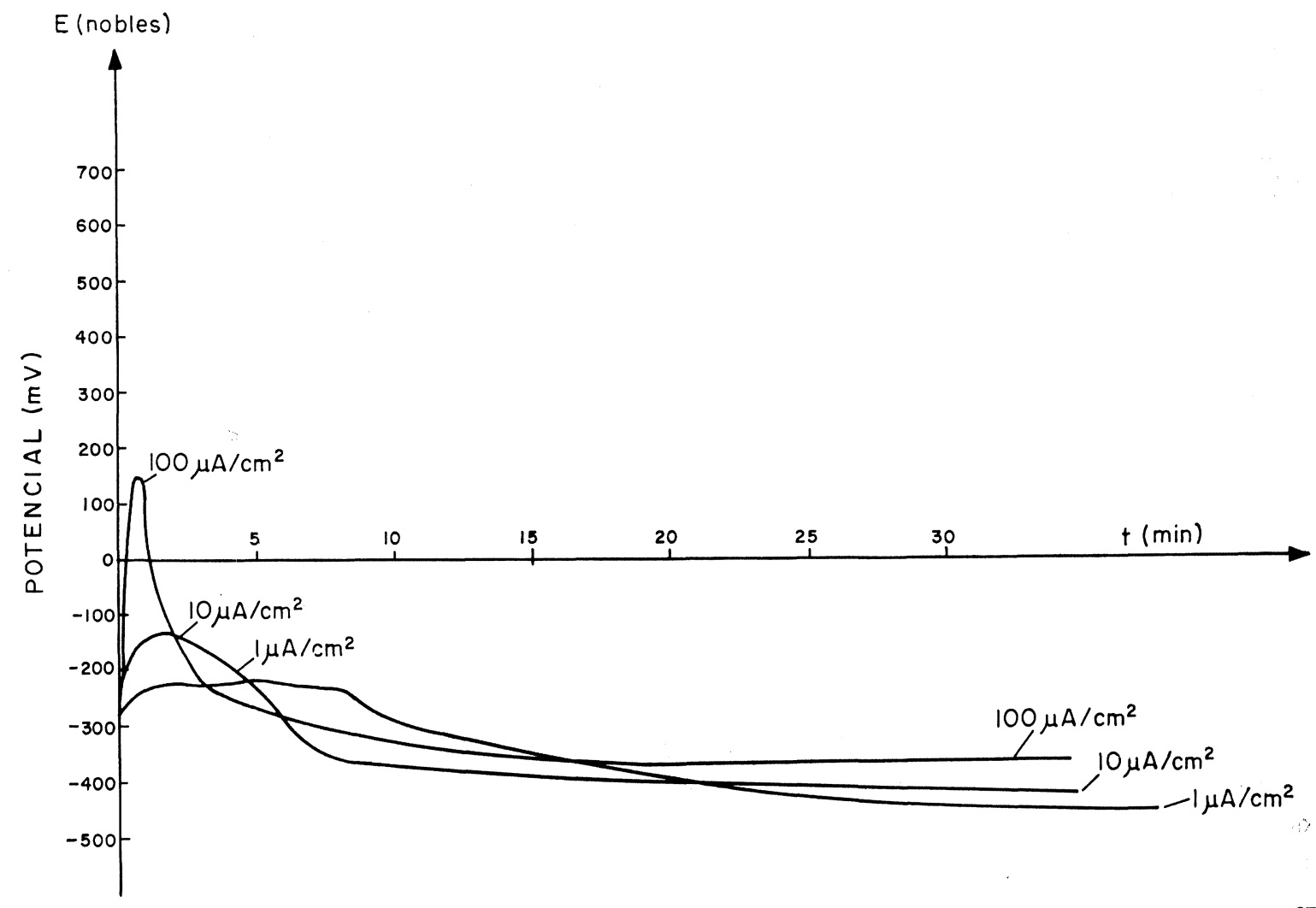


La figura 6 muestra el comportamiento de los electrodos en solución libre de cloruros y con 0,01 y $0,10 \% \mathrm{Cl}^{-}$, utilizando el método potenciostático y empleando para cada medida una probeta nueva. El descenso continuo de la intensidad a un potencial dado indica la ausencia de picaduras, y su aumento, frecuentemente después de pasar por un mínimo, la rotura de la capa de pasivación. En ausencia de $\mathrm{Cl}^{-}$se llega a potenciales de 500 y $600 \mathrm{mV}$ (a la zona transpasiva) sin detectar ningún $E_{\mathrm{r}}$. En cambio, con un $0,10 \% \mathrm{Cl}^{-}$. pequeñas polarizaciones anódicas provocan ya la ruptura de la capa de pasivación, mientras que pequeñas polarizaciones catódicas preservan a veces de la aparición de picaduras (figura 6b), y, otras las permiten. Parece, pues, que la concentración primera de cloruros es capaz de hacer descender el $E_{\mathrm{r}}$ por debajo del $E_{\text {corr }}$, de forma que pueden desarrollarse picaduras en el sistema en ausencia de toda influencia externa.

No ocurre lo mismo cuando la cantidad de cloruros es solo del $0,01 \%$, porque entonces, como se aprecia en la figura $6 \mathrm{c}, E_{\mathrm{r}}$ está situado entre $0 \mathrm{y}+40 \mathrm{mV}$ (con respecto al electrodo de calomelanos saturado) y, al ser $E_{\text {corr }}<<E_{\mathrm{r}}$, el sistema sólo podrá atacarse por picaduras bajo el efecto de algún factor que eleve sustancialmente $E_{\text {corr }}$, o rebaje el valor de $E_{\mathrm{r}}$.

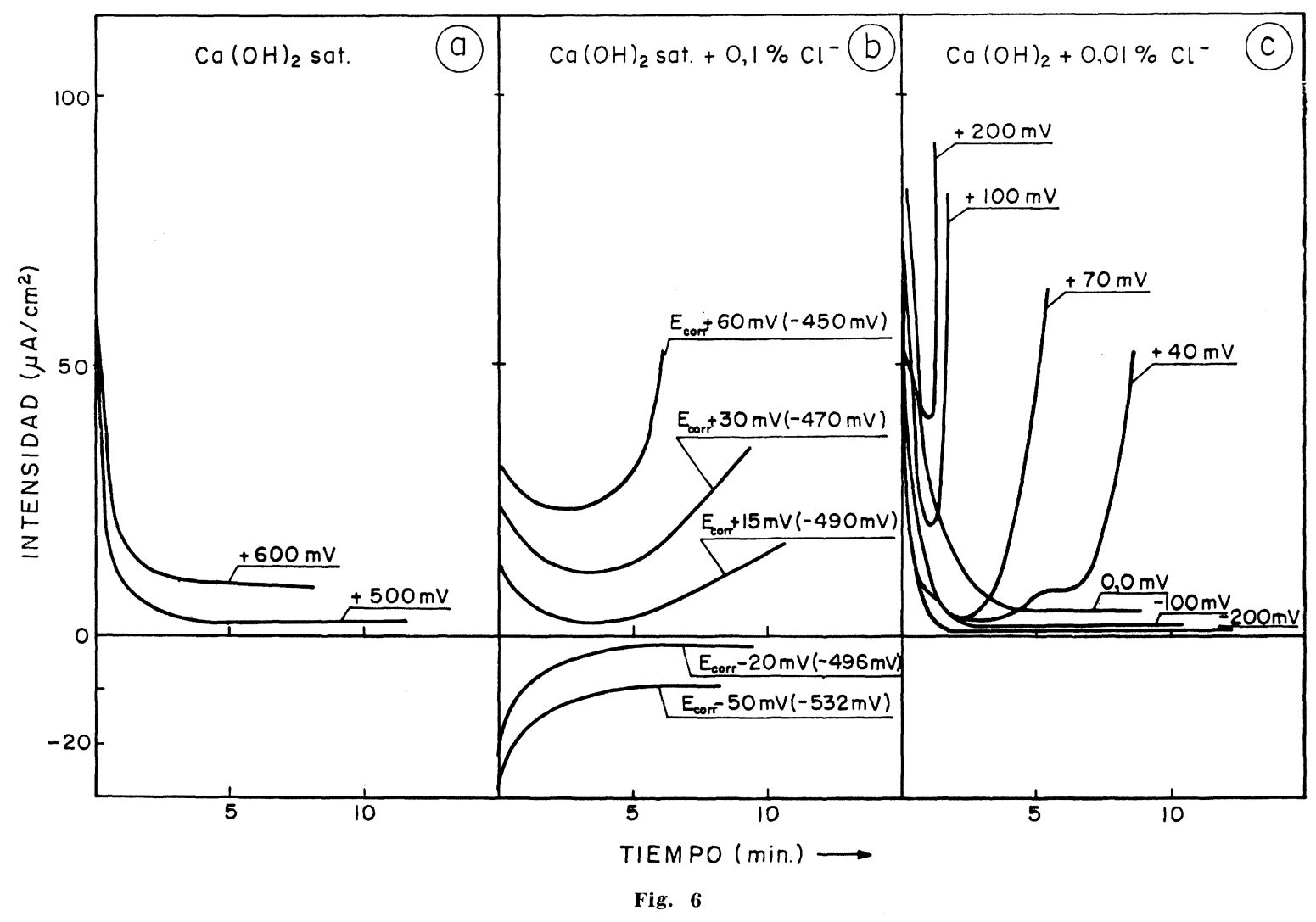

Finalmente, se muestran en la figura 7 dos curvas de polarización realizadas sobre electrodos embebidos en probetas de mortero de cemento Portland 450, fabricadas hace más de 4 años y conservadas en un interior. La línea continua corresponde a la probeta que no contiene aditivos y la de trazos a mortero al que se adicionó un $2 \% \mathrm{CaCl}_{2}$. 


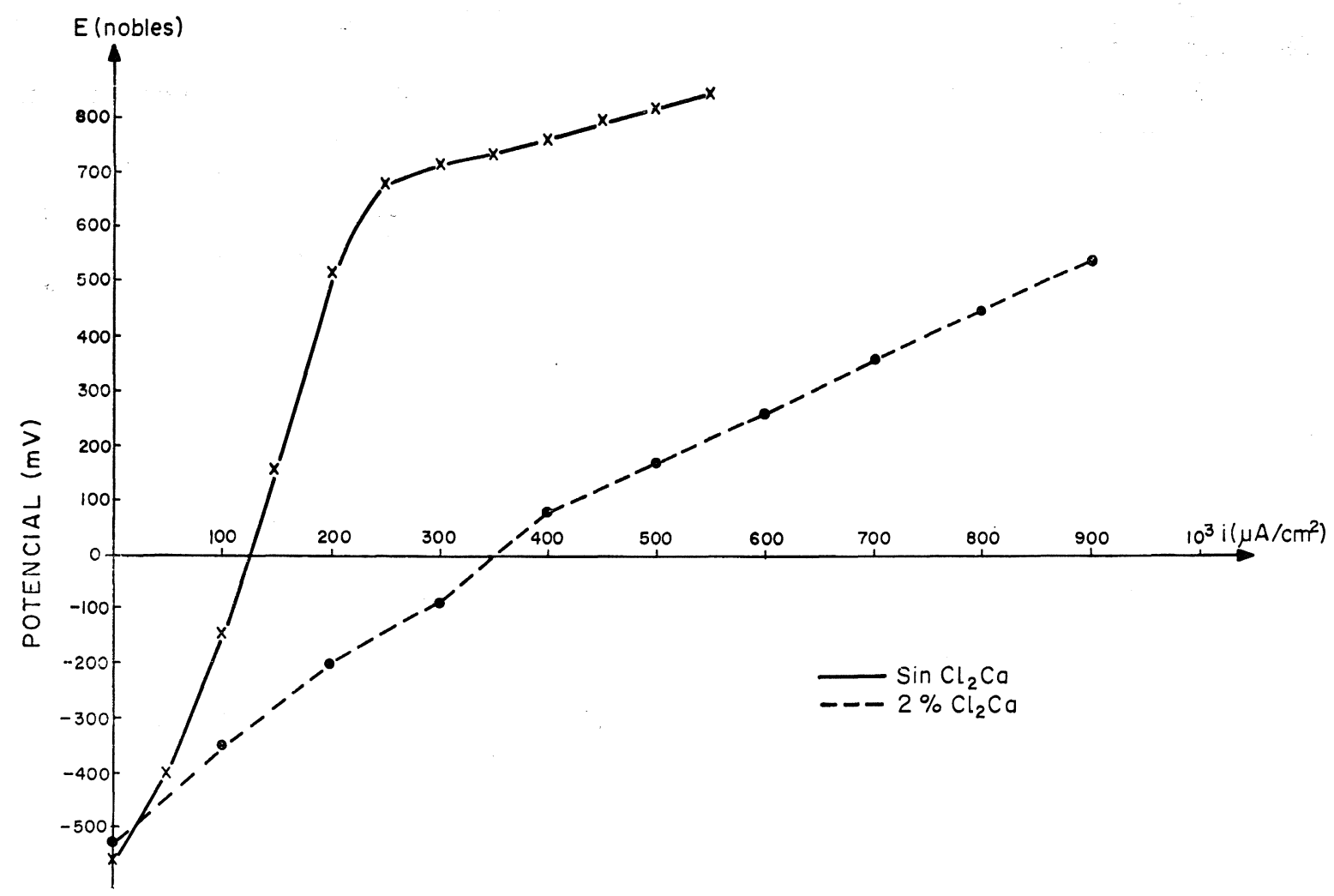

Fig. 7

Las probetas se introdujeron en disolución saturada de $\mathrm{Ca}(\mathrm{OH})_{2}$ en el momento de realizar la medida, con el fin de que la caída óhmica debida a la elevada resistividad es$p$ ミcífica del hormigón, que los aparatos de medida no pueden contrarrestar automáticamente, fuera lo menor posible.

A igualdad de polarización, se observa una gran diferencia entre la intensidad que pasa por las probetas de mortero con y sin adición de $\mathrm{CaCl}_{2}$. La falta de un potencial de ruptura anterior a la zona transpasiva, cuando no existen cloruros en el medio y, el rápido incremento de la intensidad a partir del $E_{\text {corr }}$ para la adición del $2 \% \mathrm{CaCl}_{2}$, evidencian la ausencia de picaduras en el primer caso y un considerable ataque en el segundo. Sin que tales indicaciones puedan proporcionar datos cuantitativos, no cabe duda de que el método potenciocinético aporta valiosas orientaciones sobre el efecto agresivo de las adiciones de $\mathrm{CaCl}_{2}$.

Las curvas de retorno, no incluidas en la figura, son típicas también de la presencia y ausencia de picaduras, según se hayan utilizado o no adiciones de $\mathrm{CaCl}_{2}$ (figs. 1 y 2).

El hecho de que el potencial de desprendimiento de $\mathrm{O}_{2}$ esté situado unos $200 \mathrm{mV}$ por encima del que cabría esperar, debe atribuirse al efecto de la caída óhmica a través del hormigón.

Expuestos los resultados y a efectos de su comparación con las experiencias contenidas en otros trabajos, se quiere hacer resaltar la influencia en ellos de los dos factores siguientes: el estado previo de la superficie metálica y el carácter destructivo de laś polarizaciones anódicas elevadas. 
El estado superficial puede condicionar el $E_{\mathrm{r}}$ en el sentido de que, cuanto más imperfecta sea la superficie metálica, más fácilmente podrá ser deteriorada la capa de pasivación y más catódico resultará el potencial de ruptura. Tal vez sea éste uno de los principales motivos de las discrepancias que aparecen entre diversos autores al citar valores para $E_{\mathrm{r}}$, así como la razón de que algunos hablen más de una banda de potenciales que de un valor crítico determinado. Las dispersiones que afectan a los valores de los potenciales críticos, obtenidos bien sea por el método potenciocinético o el galvanostático, pueden atribuirse, al menos en parte, a diferencias en el estado superficial de la probeta, sobre todo si se considera que el pulido mecánico aplicado consigue estados superficiales menos perfectos y reproducibles que el de tipo electrolítico (10).

Por otra parte, se ha comprobado en los ensayos efectuados que las polarizaciones anódicas superiores a $20-50 \mathrm{mV}$ alteran el estado de la probeta, tanto más, como es obvio, cuanto mayores y más duraderas sean. Así pues, el electrodo que ha sufrido una polarización anódica no tiene por qué comportarse como otro completamente nuevo. De los resultados obtenidos por medidas sucesivas a lo largo del tiempo sobre una misma probeta, al compararlos con los obtenidos utilizando un electrodo nuevo para cada ensayo, parece deducirse que, si el electrólito es susceptible de provocar ataque localizado, cada polarización anódica produce nuevas picaduras, y que en caso contrario, con un electrólito no agresivo, la polarización influye fortaleciendo la película de pasivación.

\section{I S C U S I O N}

\section{Métodos electroquímicos utilizados y sus aportaciones}

\section{Método potenciostático}

En coincidencia con diversos autores que lo han utilizado, parece ser el método que proporciona los valores más exactos para los potenciales críticos. Presenta el inconveniente de que exige el empleo de una probeta nueva para cada medida, mientras que los métodos restantes determinan los dos potenciales críticos de picado con una sola probeta.

Algunos autores afirman que cuando a una sola probeta se aplican potenciales crecientes sucesivos, esperando en cada medida el tiempo necesario para que se estabilice la intensidad, se llega a unos valores fiables de $E_{\mathrm{r}}$ y $E_{\mathrm{p}}$; pero es discutible que sea cierto para el caso que nos ocupa, ya que, si se considera que las polarizaciones anódicas superiores a $20-50 \mathrm{mV}$ pueden generar ya picaduras cuando hay cloruros presentes, es de suponer que el electrodo sometido a polarizaciones sucesivas no se comporte igual que uno nuevo.

\section{Método potenciocinético}

Ya se ha mencionado que proporciona valores de $E_{\mathrm{r}}$ y $E_{\mathrm{p}}$ afectados de grandes dispersiones, dependiendo de varios factores, esencialmente de la velocidad de polarización (figura 3). No obstante resulta valioso porque permite distinguir si existe o no susceptibilidad a la corrosión por picaduras. 
En este método es preciso considerar no sólo la curva de ida, sino también de retorno, porque, según que la intensidad de ésta para idéntico potencial sea inferior o superior a la correspondiente a aquélla, es un signo claro de fortalecimiento de la película de pasivación o de la destrucción de la misma por formación de picaduras. En alguno de los ensayos realizados con un $0,01 \% \mathrm{Cl}^{-}$no se encontró ningún $E_{\mathrm{r}}$ en la curva de ida y, sin embargo, en la de retorno se apreciaba claramente la existencia de picaduras. Todo ello confirmado posteriormente por la observación visual de la probeta. La causa de este comportamiento radica probablemente en la existencia de un período de incubación, necesario para la aparición de picaduras, mayor que el requerido para trazar la curva de i.da (potenciales crecientes).

\section{Método galvanostático}

Puede afirmarse de él, lo mismo que del potenciocinético, que es válido para comprobar si un determinado medio es capaz de producir o no ataque localizado, pero los valores hallados de los potenciales críticos pueden ser distantes de los reales, como los autores han comprobado en ocasiones, sobre todo en $E_{\mathrm{r}}$.

El valor del máximo, asimilado por algunos a $E_{\mathrm{r}}$, está fuertemente condicionado por variables cinéticas como la magnitud de la densidad de corriente aplicada (fig. 5) y el tiempo de incubación de las picaduras, por lo que, si bien su aparición revela la de picaduras, su posición no tiene relación con el valor $E_{\mathrm{r}}$ fijado por la termodinámica.

En cuanto al potencial estacionario final, alcanzado después de producida la ruptura de la capa de pasivación, se ha constatado que corresponde a un estado activo; porque, dependiendo del medio y la polarización aplicada, se encuentra unas veces por encima de $E_{\mathrm{r}}$, ya que se siguen produciendo nuevas picaduras (caso del $0,10 \% \mathrm{Cl}^{-}$); o bien se halla comprendido entre $E_{\mathrm{r}}$ y $E_{\mathrm{p}}$, pues sólo se percibe propagación de las ya existentes (caso del $\left.0,01 \% \mathrm{Cl}^{-}\right)$. Ahora bien, al permanecer el electrodo en estado activo, la intensidad aplicada ejercerá escasa influencia en su potencial, de modo que el valor estacionario final, sin coincidir con el verdadero $E_{\mathrm{p}}$, como sugieren ciertos autores, será próximo a él y, como puede comprobarse en la figura 5, está sujeto a una menor dispersión de valores que el máximo de la curva (hipotético $E_{\mathrm{r}}$ ).

En consecuencia, si se persigue obtener unos valores fiables de $E_{\mathrm{r}}$ y $E_{\mathrm{p}}$ debe recurrirse al método potenciostático, mientras que para un estudio exploratorio o para decidir si el metal o aleación es susceptible de atacarse por picaduras en el medio agresivo, basta, la mayoría de las veces, un solo ensayo por el método potenciocinético o galvanostático o, en caso de duda, la utilización conjunta de ambos.

\section{Efecto de los aditivos despasivantes e inhibidores sobre los potenciales críticos}

Ya en 1959 Kaesche y Baümel utilizaron los métodos potenciocinético y galvanostático para comprobar si determinados aditivos para el hormigón atacaban o no las armaduras. Posteriormente han abundado en el tema otros autores (21-27) y, aunque no se relacionan los resultados obtenidos con los potenciales críticos de picado, sus esfuerzos suponen aportaciones muy valiosas.

Aunque en el presente trabajo sólo se ha analizado el efecto de los iones $\mathrm{Cl}^{-} \mathrm{y} \mathrm{NO}_{2}^{-}$, es precisamente en este campo, de la posibilidad de ataque localizado a las armaduras por los aditivos para el hormigón existentes en el mercado español, en el que se proponen los autores profundizar en sucesivos ensayos, con el fin de llegar a establecer la agresividad 
potencial de un determinado aditivo o adición hacia el acero de las armaduras, para lo cual quizás deban complementarse Ios métodos electroquímicos aquí descritos con aquéllos otros que, como la medida de la resistencia de polarización, permitan una estimación cuantitativa de la velocidad de corrosión (28).

Prescindiendo de estos últimos resultados, de los expuestos anteriormente se deduce que, en disolución saturada de $\mathrm{Ca}(\mathrm{OH})_{2}$ sin cloruros, el acero permanece perfectamente protegido. Un $0,01 \% \mathrm{Cl}^{-}$puede o no provocar picaduras, haciendo sospechar que esta concentración está próxima al límite capaz de actuar como despasivante, pasando entonces a dessempeñar el estado superficial del acero un papel fundamental. Sin embargo, un 0,10 \% $\mathrm{Cl}^{-}$siempre provoca picaduras desde ios primeros momentos, independientemente del grado de acabado superficial.

En cuanto a las proporciones de $\mathrm{NO}_{2}^{-}$utilizadas, ninguna de ellas inhibió completamente el ataque de las dos proporciones de $\mathrm{Cl}^{-}$, pero tanto por el método potenciocinético como por el galvanostático se podía apreciar (fig. 4) una atenuación del ataque localizado y una constante competencia entre la acción agresiva de unos iones e inhibidora de los otros. Sin embargo, otros autores han encontrado que relaciones $\mathrm{Cl}^{-} / \mathrm{NO}_{2}^{-}$de $1 / 1$ ya evidencian una perfecta inhibición del ataque del acero (22) (23). Por todo ello, se pensó que si en el caso presente relaciones $1 / 5$, mucho más favorables que la mencionada, no se mostraban protectoras, podía deberse a que el $\mathrm{NO}_{2}^{-}$sólo se mostrase activo entre ciertos márgenes de potencial, perdiendo su efecto inhibidor a polarizaciones anódicas elevadas, por lo que no se puede detectar con los métodos electroquímicos que requieran tales polarizaciones. De hecho, comprobaciones realizadas por el método potenciostático, con polarizaciones anódicas de tan solo $20 \mathrm{mV}$, evidenciaron la aparición de picaduras cuando se adicionó un $0,10 \% \mathrm{Cl}^{-}$a la solución saturada de $\mathrm{Ca}(\mathrm{OH})_{2}$ y la ausencia de ataque localizado con un $0,10 \% \mathrm{Cl}^{-}+0,10 \% \mathrm{NO}_{2}$. La observación visual de las probetas, tras una semana de inmersión, se mostró totalmente concordante con lo acabado de expresar.

\section{Significado del potencial de corrosión}

Existe la creencia bastante difundida, apoyada por algunos trabajos sobre el tema, de considerar el $E_{\text {corr }}$ (a corriente nula) como indicativo del estado de deterioro del electrodo en el sistema metal/medio que se considere, concretamente, respecto de la corrosión de armaduras, se piensa que cuanto más noble es el potencial menos corroído estará el acero y viceversa. Esta afirmación, es cierta mientras no haya iones despasivantes en el medio, pues entonces, cuanto más noble sea el potencial, más perfecta será la película de pasivación y menor será la corrosión del electrodo. Ahora bien, si dichos iones despasivantes están presentes, un potencial muy positivo (anódico) indicará, contrariamente, una gran probabilidad de que aparezcan picaduras; y un potencial muy negativo (catódico), un riesgo escaso, o bien, que el electrodo en cuestión ha sufrido ya ataque localizado.

En resumen, dado el estado actual de conocimientos sobre la influencia del contenido en $\mathrm{Cl}^{-}$y otros iones agresivos sobre los potenciales críticos de picado del acero embebido en hormigón, el valor del potencial de corrosión no puede servir de base para un dictamen sobre el estado real de deterioro de las armaduras. 


\section{A G R A D E C I M I E N T O}

Los autores agradecen al Departamento de Corrosión y Protección del CENIM las facilidades concedidas para la realización del presente trabajo.

\section{B I B L I O G R A F I A}

(1) M. Pourbaix: Rapport Technique de CEBELCOR. R. T. n. 204 (1973).

(2) M. Janik-Czachor, A. Szummer y Z. Szklarska-Smialowska: Corr. Sci; 15 (1975), 775.

(3) A. Pourbaix: Corrosion; 27 (1971), 449.

(4) T. Suzuki, M. Yamabe e Y. Kitamura: Corrosion; 29 (1973), 18.

(5) M. MareK y R. Hochman: Corrosión; 26 (1970), 5.

(6) M. Stern y A. L. Geary: J. Electrochem. Soc; 104 (1957), 56.

(7) D. A. Jones y N. D. Greene: Corrosion; 22 (1966), 198.

(8) M. Prazak: Werk. u. Korr; 25 (1974), 104.

(9) C. Andrade y J. A. Gonzalez: 1. ${ }^{\text {er }}$ Cong. Nac. de Corr. y Prot. Madrid, junio de 1972.

(10) M. JANik-Czachor, G. H. WAgner y A. Desestret: Corr. Trait. Prot. Fin: agosto-septiembre (1971), 249.

(11) H. BöHNI: Werk. u. Korr; 25 (1974), 97.

(12) G. Herbsleb: Werk. u. Korr; 26 (1975), 929.

(13) H. Grubitsch, H. Miklautz y F. Hilbert: Werk. u. Korr; 21 (1970), 405.

(14) G. Herbsleb: Werk. u. Korr; 24 (1973), 763.

(15) Z. Szklarka-Smialowska y M. Janik-Czachor: Corr. Sci; 11 (1971), 901.

(16) Z. Szkalarska-Smitalowska: Brit. Corr. J; 10 (1975) n. 1 p. 11.

(17) J. A. Gonzalez y J. Fullea: Corr. y Protc: 5 (1974), 345.

(18) M. PourbalX y colaboradores: Corr. Sci : 3 (1963), 217.

(19) H. KAESCHE: Zement-Kalk-Gips; Vol. 12 n. 7 (1959), 289.

(20) A. BaÜMEL: Zement-Kalk-Gips; Vol. 12 n. 7 (1959), 294.

(21) K. S. Rajagopalan, N. S. Rengaswamy y T. M. Balasubramanian: J. Scient. Ind. Res; 28 (1969), 382.

(22) W. K. Gouda y G. E. Monfore: J. PCA. Res. and Dev. Lab; 7 (1965), 24.

(23) V. Amicarelli y R. Caramazza: L'Ind. Ital. del Cemento; febrero (1968), 67.

(24) K. KishitanI: Jour of the Faculty of Eng. Univ. of Tokyo (1970).

(25) J. Olden y H. Polster: Bauplanung-Bautechnik; (1974), 138.

(26) V. K. Gouda, M. A. Shater y R. Sh Mikhail: Cement and Concrete Res; 5 (1975) n. 1 p. 1.

(27) Z. Scislewski: Cahiers du Centre Scientifique et Technique du Bâtiment n. 165 diciembre 1975.

(28) C. Andrade: Tesis doctoral Universidad Complutense de Madrid, junio de 1973. 